\title{
Influence of Gap Size and Position within Gaps on Light Levels
}

\author{
Benoit Gendreau-Berthiaume and Daniel Kneeshaw \\ Département des Sciences Biologiques, Centre D'étude de la Forêt, Université du Québec à Montréal, \\ Succursale Centre-Ville, Case Postale 8888, Montréal, QC, Canada H3C 3P8 \\ Correspondence should be addressed to Benoit Gendreau-Berthiaume, ti_ben25@hotmail.com
}

Received 25 May 2009; Accepted 28 July 2009

Recommended by Andrew Gray

The previous studies have reported maximum light levels at different positions within gaps but many of these studies are based on gaps of different size. The objective of this study is to evaluate the influence of gap size and position within gap on light distribution in gaps and under the canopy north of gaps in a mixedwood temperate forest. For three gap sizes, with gap widths ranging between 0.5 and 1.5 times the height of the surrounding stand, light was measured at different positions along the north-south axis in each gap using two different techniques (hemispherical photographs and instantaneous measurements). In small gaps, the position with the most light was close to the northern edge although not under the canopy north of the gap. For both methods, the position with the highest light level shifted from the north towards the center of gaps as gap size increased which clarifies some of the variability in light measurements previously observed in gap studies. Higher light levels in the northern part of small and medium gaps compared to the southern portion could allow management of a mixture of species with intolerant species in the northern portions of gaps and tolerant species to the south.

Copyright () 2009 B. Gendreau-Berthiaume and D. Kneeshaw. This is an open access article distributed under the Creative Commons Attribution License, which permits unrestricted use, distribution, and reproduction in any medium, provided the original work is properly cited.

\section{Introduction}

Gap formation plays an important role in forest dynamics [1, 2]. Light levels increase after gap creation [3-6] and influence tree species germination, growth, survival, and consequently forest succession [7-10]. Whitmore [11] classified species into two general groups depending on light requirements: pioneer species that cannot survive in the shade and with seeds that germinate only when exposed to direct sun light for at least part of the day, such as in canopy gaps, and climax species that can germinate beneath the forest canopy and whose seedlings can survive in shade. Other authors have focused on the role of light in affecting the establishment and growth of seedlings [12-15].

Light is, however, not distributed equally in gaps. Factors such as latitude, gap size, and the canopy height surrounding the gap influence light distribution in gaps [16]. These factors consequently influence the spatial and temporal establishment of shade-tolerant and shade-intolerant species found inside gaps [17]. To consider the influence of canopy height on light condition within gaps, the ratio of gap diameter to the average height of the surrounding canopy ( $D / H$ ratio) has been used $[4,8,16]$. As the $D / H$ ratio of gaps increases, a relationship of increasing light levels and number of shade intolerant species is expected [18].

Many studies measuring light in gaps demonstrate that light distribution is not homogeneous and that the principal light gradient in temperate regions is along the north-south axis $[3,6,16]$. However, these studies used different techniques to either predict or measure different light distributions. Canham et al. [16] and Ban et al. [19], for example, developed models that predicted that the highest light levels occur under the canopy north of gaps in northern latitudes. However, a field study conducted in one of the regions modelled by Canham et al. [16] measured the highest light levels within the northern limit of the gaps [4]. Two unrelated studies in northern latitudes also found contrasting results by measuring or predicting the highest levels of light just north of the center of gaps $[3,6]$. 
The objective of this study is to examine the influence of gap size on the distribution of light along the north-south axis in the gap and to the north of the gap under the forest canopy as this will affect species recruitment. We ask the following questions: At what position will the highest levels of light be found in gaps and will this position vary with gap size? At what position in gaps do light levels differ from under closed canopy? Answers to these questions will permit us to use knowledge on plant species responses to light levels to determine optimal gap sizes to be used in forest management to favour a given species.

\section{Methods}

2.1. Study Area. The study site was located in the Duschesnay forest in central Quebec $\left(46^{\circ} 56^{\prime} \mathrm{N}, 071^{\circ} 40^{\prime} \mathrm{W}\right)$ an experimental research forest of the Quebec Ministry of Natural Resources and Wildlife. The mean annual temperature is $3.6^{\circ} \mathrm{C}$ and the mean annual precipitation falling as rain and snow is $990 \mathrm{~mm}$ and $350 \mathrm{~mm}$, respectively. The growing season varies between 150 and 170 days and occurs from May to mid September. Soils in this forest are mostly comprised of tills greater than $1 \mathrm{~m}$ in depth. The average height of the canopy was $18.75 \mathrm{~m}$ (ranging from $15.4 \mathrm{~m}$ to $23.8 \mathrm{~m}$ ). Nine prism measurements were taken to determine the mean total basal area $\left(22.4 \mathrm{~m}^{2} /\right.$ ha ranging from $14 \mathrm{~m}^{2} /$ ha to $\left.36 \mathrm{~m}^{2} / \mathrm{ha}\right)$. The mixed composition forest is dominated by yellow birch (Betula alleghaniensis) (42\% of total BA) with some sugar maple (Acer saccharum) (16\% of total BA). The conifers present in this forest were balsam fir (Abies balsamea) $(20 \%$ of total BA) and some white spruce (Picea glauca) (9\% of total BA). Other species present were American beech (Fagus grandifolia) ( $8 \%$ of total BA) and red maple (Acer rubrum) ( $5 \%$ of total BA).

2.2. Gap Selection. Gaps selected for this study consisted of manually felled gaps that were two to four years old at the moment of sampling for this study. Special care was made during logging to maintain the surrounding canopy. Gap boundaries were defined using the vertical projection of the canopy (vertically projected gap described in Ban et al. [19]). We evaluated the light gradient from the south to the north of gaps. Since the shape of gaps and the slope also influence the light gradient, we considered gaps located on level ground and gaps that were as symmetrical as possible from east to west to reduce unintended variation in light along the north-south gradient. Competing species such as mountain maple (Acer spicatum) and raspberry (Rubus idaeus) also occurred within gaps although, given the young age of the gaps, they were lower than the height $(1.5 \mathrm{~m})$ at which the light measurements were made. The canopy surrounding selected gaps was representative of the density of the forest and was without other openings for a distance equivalent to at least one tree height.

To classify gap size, the ratio of gap diameter to the canopy height $(\mathrm{D} / \mathrm{H}$ ratio) was used. This ratio was calculated by measuring the distance between the south and the north of the vertically projected gap as well as by measuring the average height of dominant trees south of the gaps since these trees had the greatest influence on the amount of light entering the gap. The height of the base of the canopy north of gaps was also measured to evaluate its influence on light levels in the understory north of gaps. An estimation of gap area was calculated based on the formula for the area of a circle. Three categories of gaps were selected for this study: small, medium, and large (Figure 1). Small gaps were those in which the diameter of the openings was less than half of the average tree height $(D / H$ ratio $\leq 0.5$, average size of $\left.51 \mathrm{~m}^{2}\right)$, medium gaps had a $D / H$ ratio of $1 \pm 0.25$, and an average size of $216 \mathrm{~m}^{2}$ while large gaps had a $D / H$ ratio equal to or larger than 1.5 and an average size of $994 \mathrm{~m}^{2}$. Gaps with a ratio between $0.5-0.75$ and $1.25-1.50$ were eliminated so that differences between each category were easier to observe. Fifteen gaps were evaluated in total, five of each gap size class.

2.3. Position of Light Measurements in Gaps and under the Forest Canopy. Light measurements were taken at equal distances from one another along the north-south axis of each gap. For all gap sizes, position 0 was the position at the southern edge of the vertically extended gap and position 1 was at its northern edge. For small gaps $(D / H$ ratio $\leq 0.5)$ five measurements were taken in each gap and one north of the gap under the adjacent forest canopy (Figure 1(a)). For medium gaps $(D / H$ ratio $1 \pm 0.25)$ seven measurements were taken in the gap and two measurements were taken under the canopy north of the gap (Figure 1(b)). Finally for large gaps $(D / H$ ratio $\geq 1.5)$ nine measurements were taken in the gap and three measurements were taken north of the gap (Figure 1(c)). The mean of 10 hemispherical photographs taken under the canopy with no gaps nearby was used to compare the light levels under closed canopy with light levels measured in gaps.

2.4. Light Measurement Methods. Two of the commonly used methods to estimate or measure light levels (hemispherical photographs and instantaneous measurements) were used at different positions in the gaps. Because hemispherical photographs provide only an estimate of potential light, a direct measurement of light (instantaneous measurements) was also used to see if similar results would be found which would thus increase the robustness of the results. The first method used was based on hemispherical canopy photographs (HPs) taken with a digital Nikon Coolpix 4500 and a fisheye lens (FC-E8 0.21x). The digital photographs were taken in June and July (thus after leaf out), under overcast conditions with uniform light to enhance the contrast between sky and canopy. The camera was levelled and oriented towards magnetic north. Images analyses were made using the Gap Light Analyser (GLA) software version 2.0. When necessary, image tools in the GLA software (contrast, brightness, gamma correct, and color plane) were used to enhance the contrast between the canopy and the sky before thresholding was applied. The resulting working images were verified thoroughly against original photographs to ensure the correct representation of the canopy. Percent of total light transmitted (Diffuse + Direct), which is the percentage of 


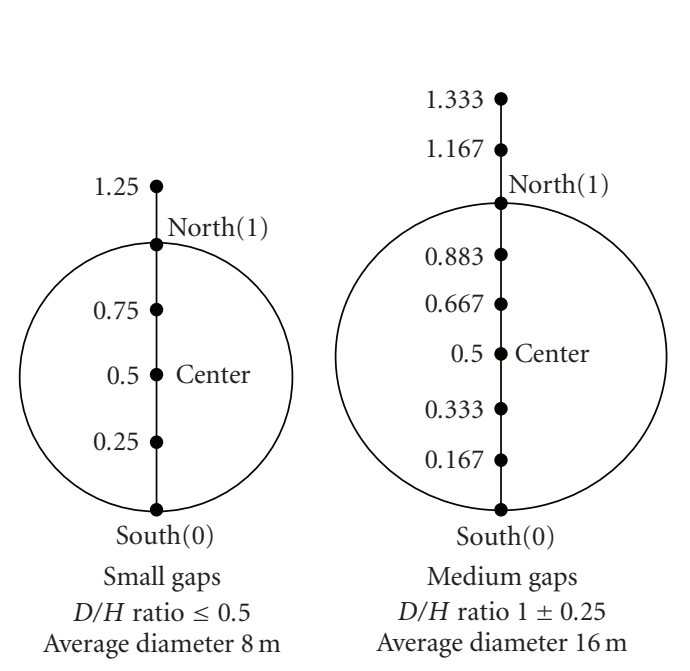

(a)

(b)

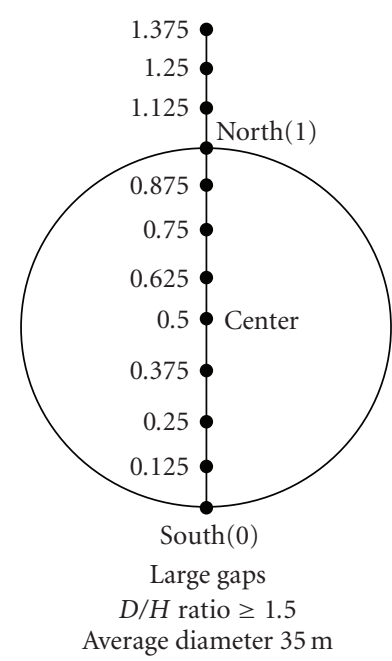

(c)

FIGURE 1: Measurement positions in gaps and under the canopy (north of gaps) for (a) small $(D / H$ ratio $\leq 0.5)$, (b) medium $(D / H$ ratio $1 \pm 0.25)$, and $(\mathrm{c})$ large $(D / H$ ratio $\geq 1.5)$ sized gaps. The average diameter of the five gaps evaluated for each category is presented to give a reference to the distance between measurement positions.

light transmitted in a completely open field and averaged for the sampling period (June and July), was determined for each photograph. The relative contribution of diffuse and direct light transmitted was assumed to be equal (50\% diffuse : $50 \%$ direct) as this has been found to be a reasonable estimate [16].

The second method used was the instantaneous measurement (IM) of the photosynthetic photon flux density (PPFD) using an LI-191 line quantum sensor (LI-COR) in gaps and a point sensor in the open. In a previous study, Messier and Puttonen [20] developed a method to estimate the \%PPFD under the canopy on completely overcast days. This method is successful under a homogeneous canopy but does not take into account the structure and spatial orientation of the canopy as well as ignoring differences in direct light [21]. In this study, measurements with the quantum sensors were made during the two hours before and after solar noon on cloudless days in the week before and after the summer solstice. This method should provide an estimation of the maximum light levels that can be found during the growing season. Every five seconds, a data logger sampled the measurements of a point sensor placed in the open and from this data; one minute averages were produced. At each gap position, two measurements were taken 15 seconds apart with the line quantum sensor oriented eastwest and again with the line quantum sensor oriented northsouth. The light value for a given position was the average of these 4 measurements. The time of each measurement was noted to compare the values of PPFD in the gaps with the values of PPFD measured in the open. From this data, the percentage of light in an open area for each position was calculated.

2.5. Data Analysis. To test whether light levels in gaps (and north of gaps) differed significantly from light levels under closed canopy, light levels measured at every location inside and north of gaps of each class size $(D / H$ ratio) were compared to light under the closed canopy using Dunnett's test. This analysis was only conducted for hemispherical photographs because measurements under closed forest canopy, used as controls in this test, were only available for this method.

Another of the questions of our study was to determine whether light levels were significantly different between positions inside and north of gaps under the canopy. Analyses of variance (ANOVA) were used to test for significant effects of location inside of the gaps on mean light levels within each size $(D / H$ ratio $)$ class. The ANOVA model included individual gaps treated as a random blocking factor and the position effect (fixed factor). In cases where the effect of position was significant $(P \leq .05)$, a Tukey-Kramer post-hoc test was used to determine which positions had significantly different light levels.

The influence of the height of canopy base on light levels measured at the first position north of gaps under the canopy was also studied. This analysis was conducted on the first position north of gaps because it was the one that would be most influenced by the height of the canopy base north of the gaps. For each method, analysis of covariance was used with a model that included height of the canopy base, gap size, and the interaction between them. In cases where gap size and the interaction (i.e., covariables) were not significant $(P<.05)$ the model was simplified to a linear regression.

Finally, for each gap size and each method, second and third degree polynomial models were fitted to the data using regression. Third degree polynomial models were a better fit to the data in all cases except for medium gaps where second degree polynomials were adopted. These regressions were used to determine the positions at which light levels were maximum (for each gap size and method) by estimating 
the position for which the model's first derivative was equal to zero. Afterward, gap size (small, medium, or large) and its interaction with the position factor were added to the model in order to test whether the distribution and hence the maximum position varied significantly between gap sizes. In the event of significant interaction, the maximum positions calculated for each gap size were compared using Tukey's HSD post-hoc test.

\section{Results}

\subsection{Light Inside and North of Gaps versus Light under Closed Canopy}

3.1.1. Hemispherical Photographs. In small gaps $(D / H$ ratio $\leq 0.5)$, light levels at the southern edge and at position 0.25 were not significantly $(P>.05)$ different from levels measured under the closed forest canopy $(16 \pm 1.8 \%$ of total light) while at the other positions, light levels were significantly higher $(P \leq .05)$ (Figure 2(a)). In medium gaps (ratio of $1 \pm 0.25)$, light levels were not significantly different $(P>.05)$ from those measured under the closed forest canopy at three positions: the two points at the southern edge (positions 0 and 0.167 ) and beyond the northern edge of the gap (position 1.333) (Figure 2(b)). Finally, in the large gaps $(D / H$ ratio $\geq 1.5)$, light levels were significantly higher $(P \leq .05)$ than those under the forest canopy at every position except the southern edge, and two positions beyond the northern edge of the gap (positions 1.25 and 1.375) (Figure 2(c)). For all gap sizes, at the first position north of the gap under the forest canopy light levels measured were significantly higher than those under the closed forest canopy (Figures 2(a), 2(b), and 2(c)).

\subsection{Comparing Light Levels at Different Positions within and North of Gaps}

3.2.1. Hemispherical Photographs (HPs). The effect of position was highly significant for each gap size (small $P=$ .0001 ; medium $P=.0016$; large $P<.0001$ ), indicating a nonuniform distribution of light inside and north of gaps. In small gaps, light levels at the northern edge and three quarters of the way through the gap towards the north (position $0.75)$ were significantly higher $(P \leq .05)$ than those close to the southern edge (positions 0 and 0.25 ) (Table $1(\mathrm{a})$ and Figure 2(a)). Light levels under the canopy north of small gaps (position 1.25) were also significantly higher than at position 0.25 . In medium gaps, there were significantly higher light levels $(P \leq .05)$ north of the center at positions 0.667 and 0.833 than those near the southern edge (positions 0 and 0.167) (Table 1(b) and Figure 2(b)). Light levels measured at the northern edge of medium gaps were also significantly higher than those at the southern edge (Table 1(b) and Figure 2(b)). Finally, in large gaps, light levels on each side of center (0.375 and 0.675) and at the center of the gaps were significantly higher than those at the southern edge and at position 0.125 (Table $1(\mathrm{c})$ and Figure 2(c)). Light levels at positions $0.75,0.875$, and at the northern edge were also

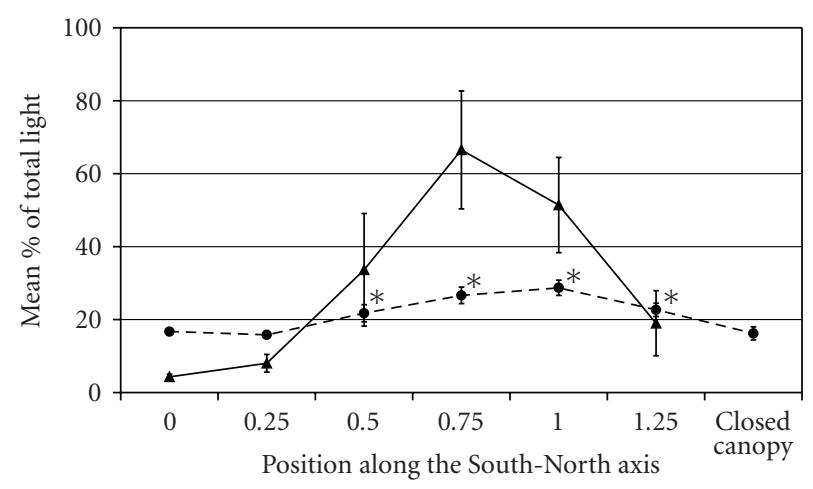

(a)

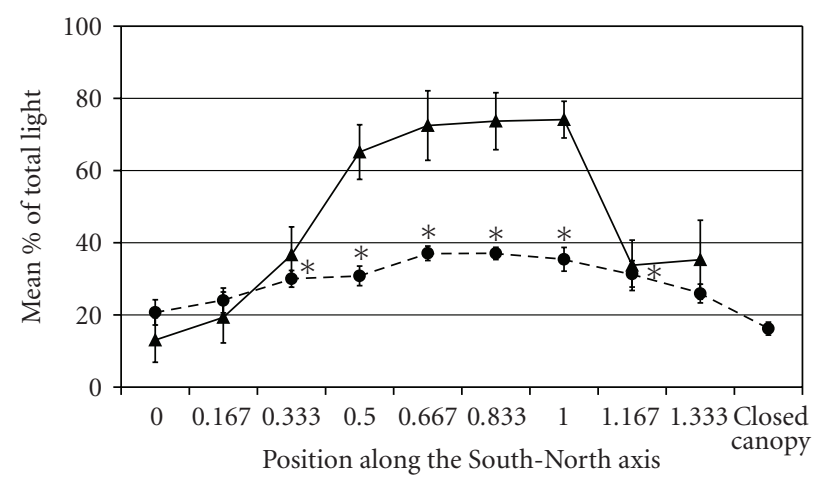

(b)

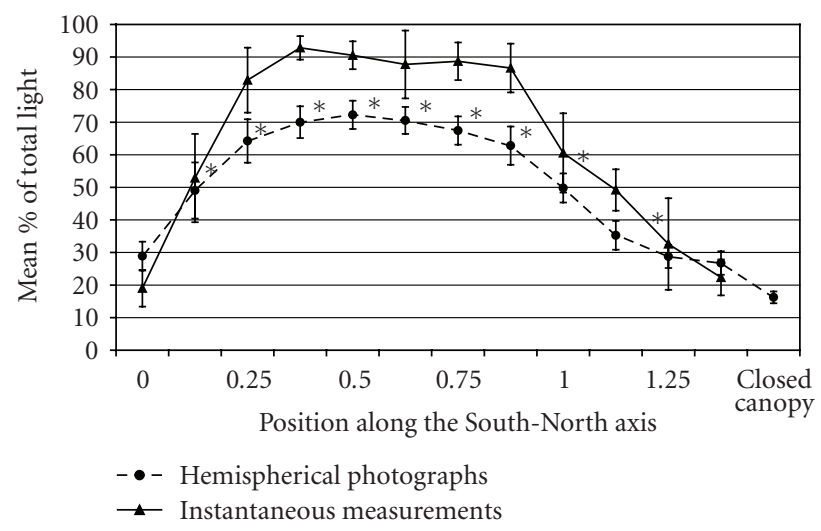

(c)

Figure 2: Mean percentage of total light relative to open conditions at different positions along the north-south axis of (a) small gaps $(D / H$ ratio $\leq 0.5$, (b) medium gaps $(D / H$ ratio $1 \pm 0.25)$, and (c) large gaps $(D / H$ ratio $\geq 1.5)$. For hemispherical photographs (circles), positions with an asterisk $(*)$ have significantly higher light levels than under closed forest canopy (Dunnett's test). Values in brackets represent standard error.

significantly higher than those at the southern edge of gaps (Table 1(c) and Figure 2(c)).

3.2.2. Instantaneous Method (IM). The effect of position was also significant for each gap size (small $P=.0004$; medium $P<.0001$; large $P<.0001)$ measured with the instantaneous light measurement methods. In small gaps, light levels were significantly higher at the northern edge and three quarters 
TABLE 1: Results of the Tukey-Kramer post-hoc test for both methods (hemispherical photographs and instantaneous measurements) and gaps of different ratio: (a) small gaps with $D / H$ ratio $\leq 0.5$, (b) medium gaps with $D / H$ ratio $=1 \pm 0.25$, and (c) large gaps with $D / H$ ratio $\geq 1.5$. Note. Positions with different letters indicate significant differences at $P<.05$.

(a) Small Gaps

\begin{tabular}{lcccccc}
\hline Methods $\backslash$ positions & 0 & 0.25 & 0.5 & 0.75 & 1 & \\
\hline Hemispherical photographs & $\mathrm{CD}$ & $\mathrm{D}$ & $\mathrm{BCD}$ & $\mathrm{AB}$ & $\mathrm{A}$ & $\mathrm{ABC}$ \\
Instantaneous measurements & $\mathrm{C}$ & $\mathrm{C}$ & $\mathrm{ABC}$ & $\mathrm{A}$ & $\mathrm{AB}$ & $\mathrm{BC}$ \\
\hline
\end{tabular}

(b) Medium Gaps

\begin{tabular}{lccccccccc}
\hline Methods $\backslash$ positions & 0 & 0.167 & 0.333 & 0.5 & 0.667 & 0.833 & 1 & 1.167 & 1.333 \\
\hline Hemispherical photographs & $\mathrm{C}$ & $\mathrm{BC}$ & $\mathrm{ABC}$ & $\mathrm{ABC}$ & $\mathrm{A}$ & $\mathrm{A}$ & $\mathrm{AB}$ & $\mathrm{ABC}$ & $\mathrm{ABC}$ \\
Instantaneous measurements & $\mathrm{D}$ & $\mathrm{D}$ & $\mathrm{BCD}$ & $\mathrm{ABC}$ & $\mathrm{AB}$ & $\mathrm{A}$ & $\mathrm{A}$ & $\mathrm{CD}$ & $\mathrm{CD}$ \\
\hline
\end{tabular}

(c) Large Gaps

\begin{tabular}{lccccccccccccc}
\hline Methods $\backslash$ positions & 0 & 0.125 & 0.25 & 0.375 & 0.5 & 0.625 & 0.75 & 0.875 & 1 & 1.125 & 1.25 & 1.375 \\
\hline Hemispherical photographs & $\mathrm{E}$ & $\mathrm{CDE}$ & $\mathrm{ABC}$ & $\mathrm{AB}$ & $\mathrm{A}$ & $\mathrm{A}$ & $\mathrm{ABC}$ & $\mathrm{ABC}$ & $\mathrm{BCD}$ & $\mathrm{DE}$ & $\mathrm{E}$ & $\mathrm{DE}$ \\
Instantaneous measurements & $\mathrm{B}$ & $\mathrm{AB}$ & $\mathrm{A}$ & $\mathrm{A}$ & $\mathrm{A}$ & $\mathrm{A}$ & $\mathrm{A}$ & $\mathrm{A}$ & $\mathrm{AB}$ & $\mathrm{AB}$ & $\mathrm{B}$ & $\mathrm{B}$ \\
\hline
\end{tabular}

of the way through the gap to the north (position 0.75 ) than those in the southern portion of the gap (positions 0 and 0.25) (Table 1(a) and Figure 2(a)). In medium gaps, light levels were significantly higher at positions $0.667,0.833$, and the northern edge (position 1.0) than those at the southern edge and position 0.167 (Table 1(b) and Figure 2(b)). Finally, in large gaps, light levels were significantly greater at each of the 6 positions around the center $(0.25,0.375$, center, $0.625,0.75$, and 0.875$)$ than those at the southern edge of gaps (Table 1(c) and Figure 2(c)). In gaps of all sizes, the results of the instantaneous method were highly variable which is consistent with Gendron et al. [22] findings that instantaneous light transmission on sunny days around noon is the most variable method of light measurement.

3.3. Influence of the Height of the Base of the Canopy. The height of the canopy base ranged from $3.3 \mathrm{~m}$ to $12.6 \mathrm{~m}$. When tested in an analysis of covariance, the covariables gap size and its interaction with height of the canopy base were not significant for either method $(P>.05)$ and thus they were dropped from the models. This indicated that the relationship between light north of gaps under the canopy and height of canopy base north of gaps never differed significantly between the different gap sizes. When tested with a linear regression, light levels measured north of gaps under the canopy increased with an increasing height of the canopy base for both methods (HP $P=.0019, y=2.19 x+$ 13.24 , and $R^{2}=0.56$; IM $P=.0003, y=4.80 x-4.92$, and $\left.R^{2}=0.67\right)$.

3.4. Light Distribution within Gaps. For both methods, the general patterns of light distribution were the same although the exact position of maximum light differed (Figure 3 and Table 2). The position with maximum light was close to the northern edge in small gaps (position 0.96 for HP and position 0.89 for IM), between the center and the northern edge in medium gaps (position 0.77 for HP and position 0.76 for IM) and at the center of the gap in large gaps (position 0.50 for HP and position 0.53 for IM) (Table 2 and Figure 3(a)). When added to the models, the interaction between gap size and position was highly significant for both methods (HP $P=.0005$ and IM $P=.0036$ ), confirming this shift in position of maximum light with gap size. Tukey's HSD post-hoc test determined that light distribution did not, however, differ between small and medium-sized gaps (HP $P=$.9979; IM $P=.6732$ ) but only between small and large gaps (HP $P=.0155$; IM $P=.0344)$ and between medium and large gaps (HP $P=.0008$; IM $P=.0007)$.

\section{Discussion}

4.1. Light under the Closed Canopy. The presence of gaps modified the light levels that were found under different places in the closed canopy and furthermore as gap size increases differences were accentuated. Ecologically in large and medium gaps the differences in light levels compared to the understory could allow for the recruitment of intolerant or mid-tolerant species while in small gaps although statistically different the absolute change in the light environment might not be sufficient to lead to differences in species recruitment. For all gap sizes, light under the forest canopy at the first position north of gaps was significantly higher than that under closed canopy forest distant from gaps (Figures 2(a), 2(b), and 2(c)).

4.2. Light Levels at Different Position and Maximum Light Levels. The two methods used gave similar patterns of light distribution (Figures 2(a), 2(b), and 2(c)). However, the instantaneous measurements provided more variable results compared to the hemispherical photographs which is consistent with a previous study comparing different light measurement methods [22]. Instantaneous measurements also measured lower light levels compared to the hemispherical photographs in low light environments (particularly in the southern part of gaps) and higher light levels in high light environments. The lower light levels measured 


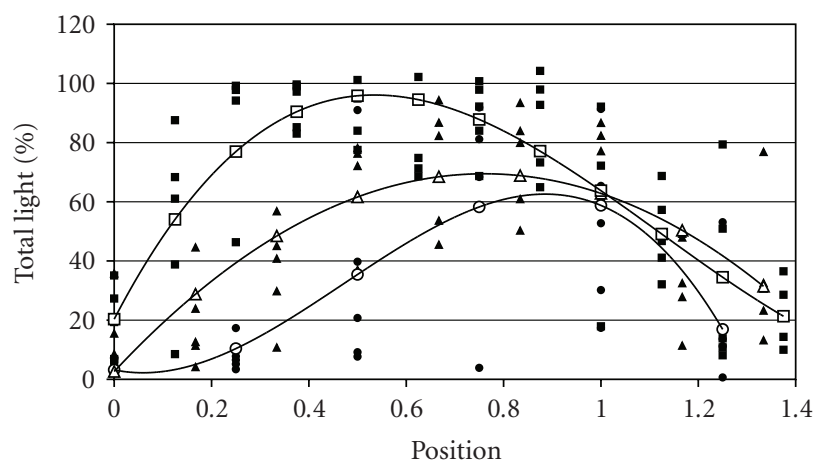

(a)

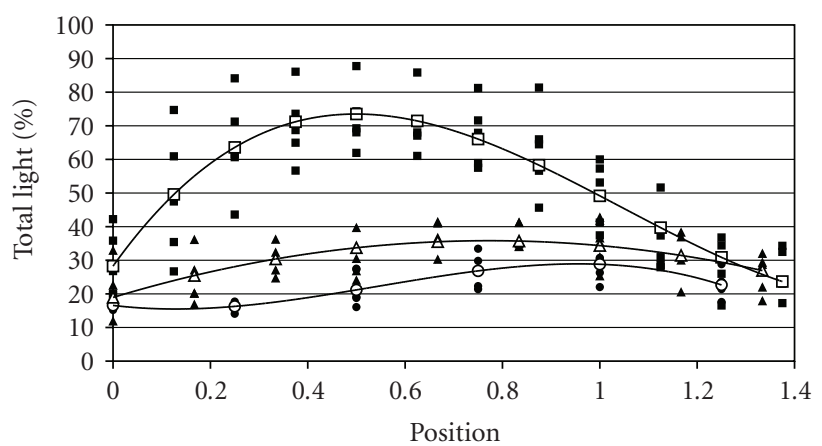

- Small gaps

- Fitted model small gap

- Medium gaps
$\Delta$ Fitted model medium gaps

- Large gaps

口 Fitted model large gaps

(b)

FIGURE 3: For each method ((a) hemispherical photographs and (b) instantaneous methods) polynomial models as described in Table 2 were fit to the data for percent of total light for each position.

TABLE 2: Results of the polynomial models for both methods (hemispherical photographs and instantaneous measurements) and for each gap size (small, medium, and large). For each model, the estimation of coefficients and their relative probability, $R^{2}$, and the position of maximum light are presented.

\begin{tabular}{|c|c|c|c|c|c|c|c|}
\hline Method & Gap size & Intercept & Position & Position $^{2}$ & Position $^{3}$ & $R^{2}$ & Maximum \\
\hline \multirow{6}{*}{ Hemispherical photographs } & \multirow{2}{*}{ Small } & 16.57 & -17.47 & 77.07 & -47.37 & \multirow{2}{*}{0.63} & \multirow{2}{*}{0.96} \\
\hline & & $P<.0001$ & $P=.1926$ & $P=.0064$ & $P=.0018$ & & \\
\hline & \multirow{2}{*}{ Medium } & 18.95 & 43.65 & -28.23 & 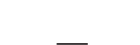 & \multirow{2}{*}{0.45} & \multirow{2}{*}{0.77} \\
\hline & & $P<.0001$ & $P<.0001$ & $P<.0001$ & & & \\
\hline & \multirow{2}{*}{ Large } & 28.26 & 202.61 & -266.84 & 85.13 & \multirow{2}{*}{0.72} & \multirow{2}{*}{0.50} \\
\hline & & $P<.0001$ & $P<.0001$ & $P<.0001$ & $P=.0007$ & & \\
\hline \multirow{6}{*}{ Instantaneous method } & \multirow{2}{*}{ Small } & 3.19 & -33.75 & 303.59 & -214.26 & \multirow{2}{*}{0.48} & \multirow{2}{*}{0.89} \\
\hline & & $P=.77$ & $P=.69$ & $P=.0804$ & $P=.0216$ & & \\
\hline & \multirow{2}{*}{ Medium } & 2.63 & 175.79 & -115.60 & & \multirow{2}{*}{0.57} & \multirow{2}{*}{0.76} \\
\hline & & $P=.7050$ & $P<.0001$ & $P<.0001$ & - & & \\
\hline & \multirow{2}{*}{ Large } & 20.32 & 316.52 & -388.88 & 115.79 & \multirow{2}{*}{0.67} & \multirow{2}{*}{0.53} \\
\hline & & $P=.0087$ & $P<.0001$ & $P<.0001$ & $P=.0075$ & & \\
\hline
\end{tabular}

by hemispherical photographs in high light environments may be due to the fact that the evaluation of light using hemispherical photographs does not take into consideration beam enrichment by reflected light as well as that transmitted by foliage [22]. The lower light levels that we observed with the instantaneous measurements for the southern part of gap may be due to the time of day ( 2 hours before and after solar noon) at which measurements were taken; since the sun would be shaded by trees at the southern end of gaps. Analysis of hemispherical photographs, on the other hand, calculates the whole spectrum of the sun's path including low angle light reaching the southern end of gaps when the sun is at its most northern positions at the beginning and the end of the day. The estimated light levels provided by hemispherical photographs are probably more important for vegetation within gaps because they represent the light conditions of the growing season at the different positions within gaps, while instantaneous methods provide an estimation of maximum light on a given day which might not be representative of overall light conditions.

The higher levels of light measured in the northern portion compared to the southern end of small and medium gaps (Figures 2(a), 2(b) and Tables 1(a), 1(b)) are due to the northern latitude of this study site where the sun angle is lower than in equatorial regions [16]. As suggested above, these differences in light levels even if significant might not be sufficient in small gaps to allow for the establishment of species of different shade tolerance. In medium and large gaps however, light levels are almost twice as high in the northern part of gaps compared to the southern part and could be sufficient to allow for the establishment of intolerant or mid-tolerant species (like Betula allenghiensis). The previous studies have observed such species partitioning within gaps in the eastern boreal forest 
[17], the north eastern US [7, 23], and the pacific northwest, while for one study also in the pacific northwest two years after harvesting gap partitioning was still not apparent [10].

In small gaps, higher light levels were also measured north of gaps under the canopy compared to in the southern part of the gaps (Figure 2(a), Table 1(a)) which is consistent with Canham et al. [16] and Ban et al. [19] reasoning. However, in this study, the position with the highest light levels was found close to the northern edge in small gaps (Figures 3(a), 3(b) and Table 2) and not under the canopy north of gaps as proposed by the previous authors. In our study, the height of the canopy base at the northern end of a gap was found to influence the light levels north of gaps under the canopy. Although this may seem intuitive it has not been commonly discussed but it could explain the difference between our study and Canham et al. [16] model as crowns of $70 \mathrm{~m}$ high Douglas fir-hemlock forest could have canopy bases that are much higher than the ones in this study (mean of $5 \mathrm{~m}$ high for small gaps).

4.3. Light Distribution in Gaps of Different Sizes. Many earlier studies $[4,6,24]$ have focused on light measurements in gaps of a given size or a small range of sizes and this has led to some of the apparent discrepancy in position of highest light. Researchers working on gaps of comparable size-whether small [4], medium [24], or large [5, 25]—have reported light to be highest at the same positions as the gaps of similar size in this study. However, our approach of measuring light across a gradient of gap sizes shows this shift in the location of the highest light towards the centre as gap size increases (Figures 3(a) and 3(b)). These results could explain why Gray et al. [4] and Canham et al. [16] found different positions of the highest light levels even if they were in the same study area and used similar methods. Closer inspection of results shows that gap sizes vary between studies (Canham et al. [16] $D / H$ ratio of 0.14-0.29; Gray et al. [4] D/H ratio of 0.2-1.0) and it can be observed that in gaps with a greater $D / H$ ratio the highest light levels were found closer to the center of the gaps.

However, it is worth noting that the interaction between factors such as latitude and gap size should also be investigated to determine how their combined influences affect light distribution in gaps. For example, in a very high latitude study site $\left(61^{\circ} 48^{\prime} \mathrm{N}\right)$ the position with maximum light levels was found to occur north of the gap center even in a very large opening with a $\mathrm{D} / \mathrm{H}$ ratio of 2.5 [3]. Maximum light levels in large sized gaps shift to gap centre as latitude decreases [5]. In small gaps light shifts even more strongly from gap centre to beyond the northern gap edge as latitude increases. Ban et al. [19] suggest that individuals might thus establish in these high light areas north of gaps where the forest is dominated by small openings and thus require multiple gap events to reach the canopy.

Our research clarifies some of the differences between earlier studies by showing that gap sizes (as measured with the $D / H$ ratio) affect the light distribution in gaps and under the canopy north of gaps. Therefore when gaps are made for management purposes in temperate regions, the size of the opening ( $D / H$ ratio) should be considered, as well as the latitude, to accurately assess which species can successfully recruit at different positions in gaps based on light levels and species shade tolerance. The difference in light levels measured north and south of medium gaps could allow the establishment of intolerant (or mid-tolerant) species in the northern portion of gaps and shade tolerant species in the southern portion of gaps. For the most intolerant species, larger gaps should be favoured due to the very high light levels found along most of the north-south gradient while for tolerant species small gaps would be more appropriate. Such reasoning could be used in the perspective of mixedwood management to ensure conditions favourable to different species $[26,27]$. However, researchers should also consider the effect of different light levels on factors such as soil moisture levels necessary for seedling germination [10]. This would follow MacDonald and Thompson's [28] recommendation for mixedwood management that different species can be separated into patches where treatments (or microhabitat in this case) are more suited to each species requirements.

\section{Acknowledgments}

This project was funded by the National Science and Engineering Research Council. A special thanks to René Dion from the Duschesnay forest for his collaboration in the field work in this project. Thanks to Julie Messier who helped with the field work and also to David Bird, Gerardo Reyes, Alain Leduc, and Stéphane Daigle for their helpful comments on the manuscript as well as the reviewers and the editor.

\section{References}

[1] J. S. Denslow and T. Spies, "Canopy gaps in forest ecosystemsan introduction," Canadian Journal of Forest Research, vol. 20, no. 5 , p. 619, 1990.

[2] W. J. Platt and D. R. Strong, "Special feature: gaps in forest ecology," Ecology, vol. 70, no. 3, p. 535, 1989.

[3] M. de Chantal, K. Leinonen, T. Kuuluvainen, and A. Cescatti, "Early response of Pinus sylvestris and Picea abies seedlings to an experimental canopy gap in a boreal spruce forest," Forest Ecology and Management, vol. 176, no. 1-3, pp. 321-336, 2003.

[4] A. N. Gray, T. A. Spies, and M. J. Easter, "Microclimatic and soil moisture responses to gap formation in coastal Douglasfir forests," Canadian Journal of Forest Research, vol. 32, no. 2, pp. 332-343, 2002.

[5] J. P. McGuire, R. J. Mitchell, E. B. Moser, S. D. Pecot, D. H. Gjerstad, and C. W. Hedman, "Gaps in a gappy forest: plant resources, longleaf pine regeneration, and understory response to tree removal in longleaf pine savannas," Canadian Journal of Forest Research, vol. 31, no. 5, pp. 765-778, 2001.

[6] E. Ritter, L. Dalsgaard, and K. S. Einhorn, "Light, temperature and soil moisture regimes following gap formation in a seminatural beech-dominated forest in Denmark," Forest Ecology and Management, vol. 206, no. 1-3, pp. 15-23, 2005.

[7] A. N. Gray and T. A. Spies, "Gap size, within-gap position and canopy structure effects on conifer seedling establishment," Journal of Ecology, vol. 84, no. 5, pp. 635-645, 1996. 
[8] A. N. Gray and T. A. Spies, "Microsite controls on tree seedling establishment in conifer forest canopy gaps," Ecology, vol. 78, no. 8, pp. 2458-2473, 1997.

[9] R. VanPelt and J. F. Franklin, "Response of understory trees to experimental gaps in old-growth Douglas-fir forests," Ecological Applications, vol. 9, no. 2, pp. 504-512, 1999.

[10] E. F. Wright, K. D. Coates, and P. Bartemucci, "Regeneration from seed of six tree species in the interior cedar-hemlock forests of British Columbia as affected by substrate and canopy gap position," Canadian Journal of Forest Research, vol. 28, no. 9, pp. 1352-1364, 1998.

[11] T. C. Whitmore, "Canopy gaps and the 2 major groups of forest trees," Ecology, vol. 70, no. 3, pp. 536-538, 1989.

[12] X. Dai, "Influence of light conditions in canopy gaps on forest regeneration: a new gap light index and its application in a boreal forest in east-central Sweden," Forest Ecology and Management, vol. 84, no. 1-3, pp. 187-197, 1996.

[13] J. S. Denslow, "Tropical rainforest gaps and tree species diversity," Annual Review of Ecology and Systematics, vol. 18, pp. 431-451, 1987.

[14] J. S. Denslow and G. S. Hartshorn, "Tree-fall gap environments and forest dynamic processes," in La Selva: Ecology and Natural History of a Neotropical Rain Forest, pp. 120-127, 1994.

[15] A. B. Nicotra, R. L. Chazdon, and S. V. B. Iriarte, "Spatial heterogeneity of light and woody seedling regeneration in tropical wet forests," Ecology, vol. 80, no. 6, pp. 1908-1926, 1999.

[16] C. D. Canham, J. S. Denslow, W. J. Platt, J. R. Runkle, T. A. Spies, and P. S. White, "Light regimes beneath closed canopies and tree-fall gaps in temperate and tropical forests," Canadian Journal of Forest Research, vol. 20, no. 5, pp. 620-631, 1990.

[17] D. D. Kneeshaw and Y. Bergeron, "Spatial and temporal patterns of seedling and sapling recruitment within canopy gaps caused by spruce budworm," Ecoscience, vol. 6, no. 2, pp. 214-222, 1999.

[18] A. H. deRömer, D. D. Kneeshaw, and Y. Bergeron, "Small gap dynamics in the southern boreal forest of eastern Canada: do canopy gaps influence stand development?" Journal of Vegetation Science, vol. 18, no. 6, pp. 815-826, 2007.

[19] Y. Ban, H. Xu, Y. Bergeron, and D. D. Kneeshaw, "Gap regeneration of shade-intolerant Larix gmelini in old-growth boreal forests of northeastern China," Journal of Vegetation Science, vol. 9, no. 4, pp. 529-536, 1998.

[20] C. Messier and P. Puttonen, "Spatial and temporal variation in the light environment of developing Scots pine stands: the basis for a quick and efficient method of characterizing light," Canadian Journal of Forest Research, vol. 25, no. 2, pp. 343354, 1995.

[21] K. J. Stadt, S. M. Landhäusser, and J. D. Stewart, "Comment: the effects of direct-beam light on overcast-day estimates of light availability," Canadian Journal of Forest, vol. 27, pp. 272274, 1997.

[22] F. Gendron, C. Messier, and P. G. Comeau, "Comparison of various methods for estimating the mean growing season percent photosynthetic photon flux density in forests," Agricultural and Forest Meteorology, vol. 92, no. 1, pp. 55-70, 1998.

[23] R. T. Busing and P. S. White, "Species diversity and small-scale disturbance in an old-growth temperate forest: a consideration of gap partitioning concepts," Oikos, vol. 78, no. 3, pp. 562-568, 1997.

[24] J. L. Gagnon, E. J. Jokela, W. K. Moser, and D. A. Huber, "Dynamics of artificial regeneration in gaps within a longleaf pine flatwoods ecosystem," Forest Ecology and Management, vol. 172, no. 2-3, pp. 133-144, 2003.
[25] D. L. Phillips and D. J. Shure, "Patch-size effects on early succession in southern appalachian forests," Ecology, vol. 71, no. 1, pp. 204-212, 1990.

[26] V. J. Lieffers and J. A. Beck, "A semi-natural approach to mixedwood management in the prairie provinces," The Forestry Chronicle, vol. 70, no. 3, pp. 260-264, 1994.

[27] G. B. MacDonald, "The case for boreal mixedwood management: an Ontario perspective," The Forestry Chronicle, vol. 71, no. 6, pp. 725-734, 1995.

[28] G. B. MacDonald and D. J. Thompson, "Responses of planted conifers and natural hardwood regeneration to harvesting, scalping, and weeding on a boreal mixedwood site," Forest Ecology and Management, vol. 182, no. 1-3, pp. 213-230, 2003. 

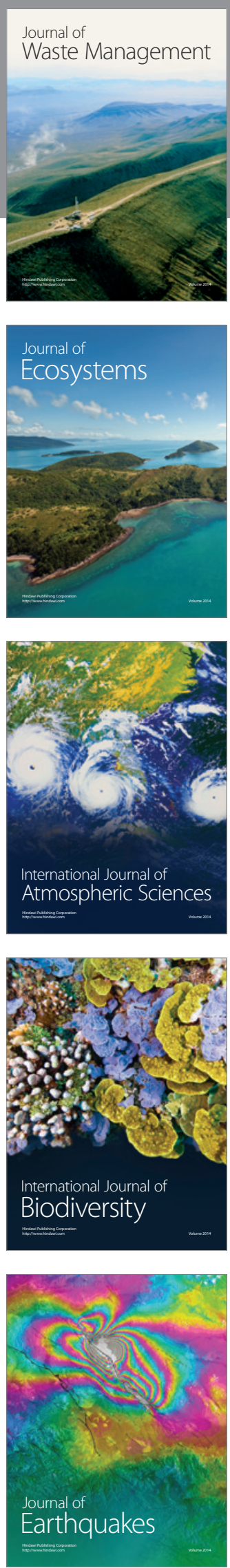
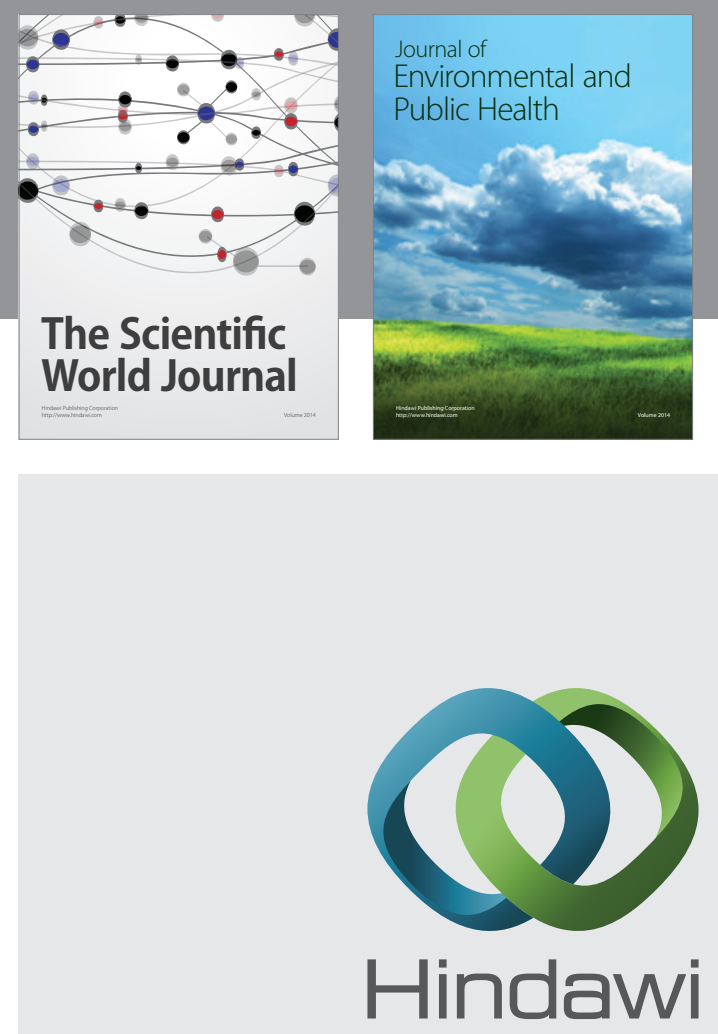

Submit your manuscripts at

http://www.hindawi.com
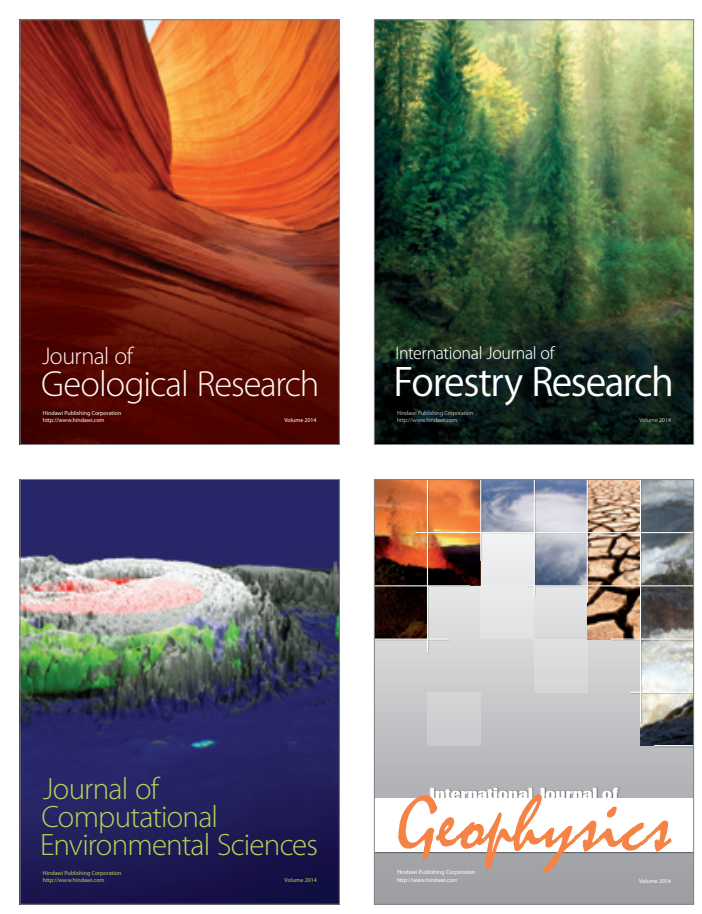
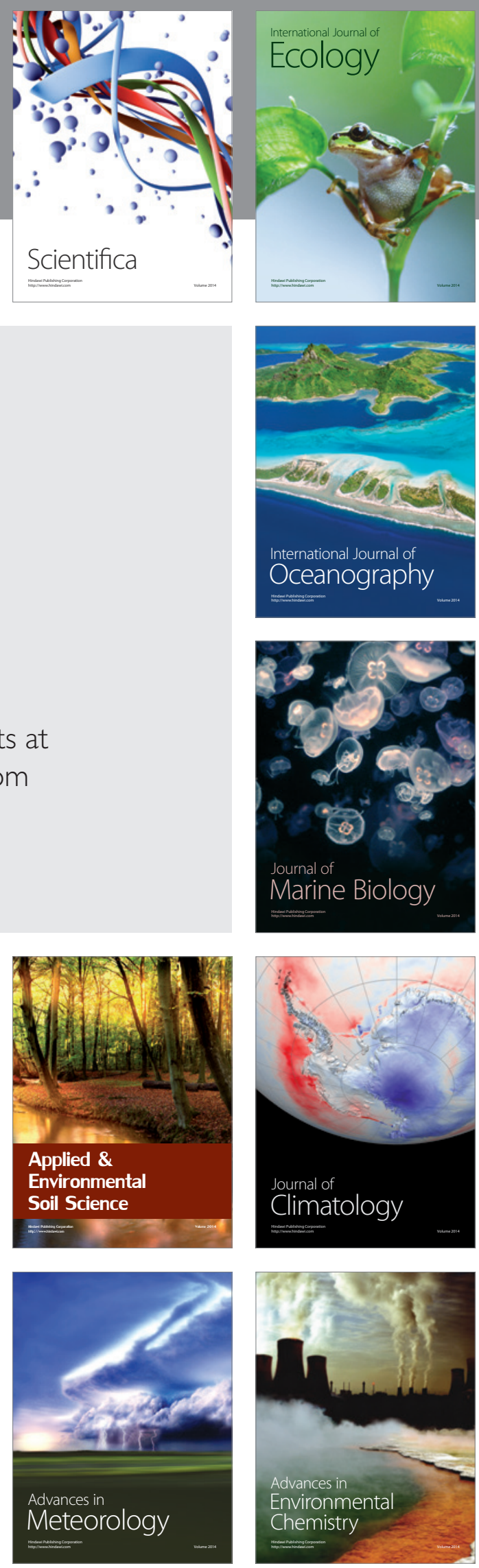